\title{
Tunable Anisotropy in Inverse Opals and Emerging Optical Properties
}

\section{Citation}

Phillips, Katherine R., Nicolas Vogel, Yuhang Hu, Mathias Kolle, Carole C. Perry, and Joanna Aizenberg. 2014. "Tunable Anisotropy in Inverse Opals and Emerging Optical Properties."

Chemistry of Materials 26 (4) (February 6): 1622-1628. doi:10.1021/cm403812y.

\section{Published Version}

doi: $10.1021 / \mathrm{cm} 403812 y$

\section{Permanent link}

http://nrs.harvard.edu/urn-3:HUL.InstRepos:37255330

\section{Terms of Use}

This article was downloaded from Harvard University's DASH repository, and is made available under the terms and conditions applicable to Open Access Policy Articles, as set forth at http:// nrs.harvard.edu/urn-3:HUL.InstRepos:dash.current.terms-of-use\#OAP

\section{Share Your Story}

The Harvard community has made this article openly available.

Please share how this access benefits you. Submit a story.

Accessibility 


\title{
Tunable Anisotropy in Inverse Opals and Emerging
}

\section{Optical Properties}

Katherine R. Phillips, ${ }^{\dagger}$ Nicolas Vogel, ${ }^{\ddagger}$ Yuhang Hu, Mathias Kolle,,$"$, Carole C. Perry, ${ }^{\S}$ and Joanna Aizenberg ${ }^{\dagger, * * *}$

'Department of Chemistry and Chemical Biology, "School of Engineering and Applied Sciences, and ${ }^{8}$ Interdisciplinary Biomedical Research Centre, Nottingham Trent University, Clifton Lane, Nottingham NG11 8NS.

KEYWORDS: inverse opals, anisotropy, photonic crystals, self-assembly, silica, colloids, optical properties

\begin{abstract}
Using self-assembly, nanoscale materials can be fabricated from the bottom up. Opals and inverse opals are examples of self-assembled nanomaterials made from crystallizing colloidal particles. As self-assembly requires a high level of control, it is challenging to use building blocks with anisotropic geometry to form complex opals, which limits the realizable structures. Typically, spherical colloids are employed as building blocks, leading to symmetric, isotropic superstructures. However, a significantly richer palette of directionally dependent properties are expected if less symmetric, anisotropic structures can be created, especially originating from the assembly of regular, spherical particles. Here we show a simple method to introduce anisotropy into inverse opals by subjecting them to a post-assembly thermal treatment
\end{abstract}


that results in directional shrinkage of the silica matrix caused by condensation of partially hydrated sol-gel silica structures. In this way, we can tailor the shape of the pores, and the anisotropy of the final inverse opal preserves the order and uniformity of the self-assembled structure, while completely avoiding the need to synthesize complex oval-shaped particles and crystallize them into such target geometries. Detailed X-ray photoelectron spectroscopy (XPS) and infrared (IR) spectroscopy studies clearly identify increasing degrees of sol-gel condensation in confinement as a mechanism for the structure change. A computer simulation of structure changes resulting from the condensation-induced shrinkage further confirmed this mechanism. As an example of property changes induced by the introduction of anisotropy, we characterized the optical spectra of the anisotropic inverse opals and found that the optical properties can be controlled in a precise way using calcination temperature.

\section{INTRODUCTION}

Self-assembly provides a way to structure materials with nanoscale dimensions without using expensive, complicated, and time consuming top-down fabrication methods. ${ }^{1}$ However, selfassembly requires a high level of control over the assembly conditions and the morphology and composition of individual unit elements..$^{2-6}$ This limits the choice of constituent building blocks that can be used and hence the variety of accessible assembly structures formed by the building blocks' minimum-free-energy arrangements. ${ }^{6-9}$ In particular, spherical colloids form highly symmetric, face-centered cubic crystals, called opals. ${ }^{10}$ This structure can be inverted to form an inverse opal by filling in the interstitial spaces and removing the colloidal template, resulting in a continuous matrix of periodically arranged, interconnected voids with the same symmetry as the original colloidal template. 
Inverse opals with high long-range order can be made using a co-assembly process. ${ }^{11} \mathrm{~A}$ thin film is grown by evaporative deposition of polymeric colloids in the presence of a silica sol-gel precursor that condenses in the colloidal crystal interstitial spaces. The co-assembly method allows the growth of extremely highly ordered crystals with single domains stretching over macroscopic dimensions. ${ }^{11}$ Upon calcination, the polymeric colloids are completely combusted and the silica precursor fuses into a dense silicon dioxide scaffold, creating a hexagonally closepacked array of interconnected voids.

Highly interconnected porosity and long-range order are two key attributes of inverse opal structures that make them valuable for a variety of applications. Due to their periodic nature, inverse opals are a versatile material platform for controlling electromagnetic ${ }^{12-14}$ and acoustic $^{15}$ wave propagation. In particular, if their periodicity is tailored to the length scale of the wavelength of visible light, inverse opals interact strongly with visible light, allowing applications in solar cells, ${ }^{16,17}$ as light emitting diodes, ${ }^{18}$ and as structural color-based coatings. ${ }^{19,20}$ In addition, inverse opals' interconnected porosity and high surface area are essential for their use in many other contexts, ${ }^{21}$ including batteries ${ }^{22,23}$ and catalysts, ${ }^{24,25}$ as well as sensors of liquid media, ${ }^{26}$ scaffolds for tissue engineering, ${ }^{27}$ substrates for omniphobic surfaces, ${ }^{28}$ and for studying diffusion processes in confinement. ${ }^{29}$ All of these remarkable properties of inverse opals depend strongly on precise control over the structural uniformity. Spherical colloids are simple to synthesize and crystallize, so they are typically used as templates. However, anisotropic structures would offer a range of unique, direction-dependent properties due to the changes in the angular periodicities and directional porosity, and are therefore highly desirable, but a simple method to obtain anisotropic inverse opals is necessary in order to investigate these directional properties. 
Prior research efforts to form ordered structures with different morphologies have primarily focused on the design and crystallization of anisotropically shaped colloidal particles. ${ }^{30-37}$ However, their synthesis is demanding and crystallization into uniform, defect-free structures remains a challenge. ${ }^{38-40}$ Successful examples include crystallization of gold nanorods, ${ }^{41-44}$ assembly of anisotropic magnetic particles in magnetic fields ${ }^{45}$ DNA templating ${ }^{46}$ of anisotropic colloidal crystals, and more recently, assembly of anisotropic silica rods in an electric field. ${ }^{47}$ These anisotropic structures have important directional properties and have been used for plasmonic sensing. ${ }^{41,44}$ Alternatively, the challenges of synthesizing and crystallizing anisotropic particles can be avoided by introducing anisotropy after the assembly of isotropic building blocks, for example by mechanical deformation of elastomeric inverse opal structures. ${ }^{13,33}$

Here we describe a simple approach to create anisotropy in inverse opal structures consisting of an inorganic, non-flexible metal oxide matrix. Our approach allows us to precisely tailor the anisotropy while preventing the need for complex crystallization techniques. Instead, we take advantage of the molecular state of the structure to introduce anisotropy. Silica sol-gels undergo condensation of remaining hydroxyl groups at high temperature, leading to volume decrease via loss of water. ${ }^{48-49}$ In the co-assembly process, the material is deposited on a substrate that holds the sol-gel material in place. This surface constraint prevents shrinking in the plane of the substrate and leads to directional out-of-plane contraction of the film, as shown schematically in cross-section in Scheme 1. This provides a facile method to introduce anisotropy into the system after assembly, resulting in controlled changes in the inverse opal properties. We present a detailed investigation of this calcination-induced anisotropy in self-assembled inverse opals. The calcination temperature controls the degree of condensation, as confirmed using X-ray photoelectron spectroscopy (XPS) and infrared spectroscopy (IR). The amount of shrinkage 
directly relates to the degree of condensation, allowing us to precisely alter the pore shape and to tailor properties that depend strongly on the pore geometry of the structure. In particular, we demonstrate how the optical properties of inverse opals change with increasing anisotropy. It is important to note that the anisotropy is generated during post-assembly processing, thus circumventing the complex synthesis and assembly of anisotropic building blocks. Instead, a generalized and reliable assembly strategy of spherical particles is used to generate crystals with adjustable pore shape anisotropy.

Scheme 1. Condensation in the silica matrix of an inverse opal causing anisotropic shrinking.

\section{Isotropic inverse opal}

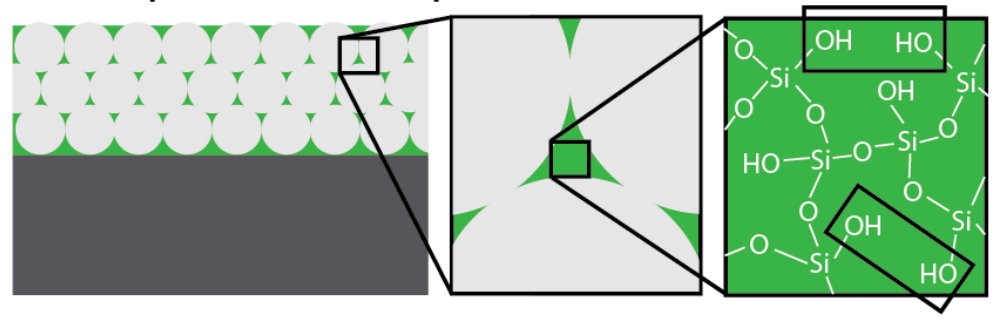

\section{Anisotropic inverse opal}

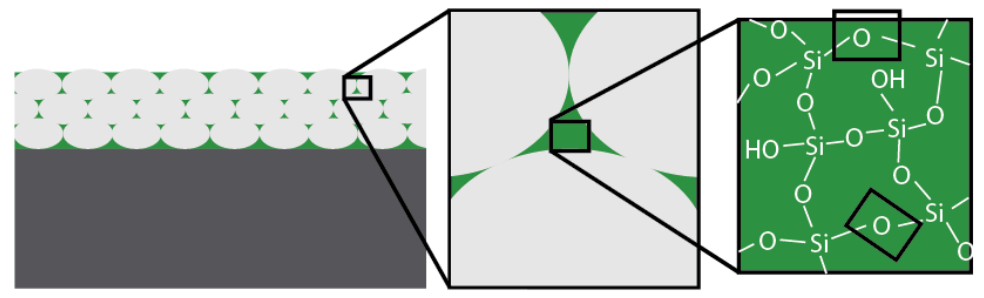

\section{EXPERIMENTAL SECTION}

Chemicals and materials. Inverse opals were synthesized using a process described in detail elsewhere. ${ }^{11}$ Briefly, polystyrene colloids with diameters of 230,330 , and $410 \mathrm{~nm}$ were synthesized using a surfactant-free emulsion polymerization process. ${ }^{50}$ They were then diluted in water to make $0.1 \%$ solid content in $20 \mathrm{~mL}$ total volume. $150 \mu \mathrm{L}$ of pre-hydrolyzed TEOS solution $(1: 1: 1.5$ by weight of tetraethyl orthosilicate : $0.1 \mathrm{M} \mathrm{HCl}:$ ethanol, stirred for $1 \mathrm{~h})$ 
was added and the mixture was sonicated. A silicon wafer was suspended vertically in the vial. The vial was then placed inside an oven for two days to allow for evaporation of the solvent, leaving behind a colloidal crystal with a silica matrix surrounding it. A Memmert UF110 oven set to $65^{\circ} \mathrm{C}$ stabilized by a pneumatic vibration-free table was used for the co-assembly process. The colloidal crystal template was removed either by submersion in toluene or tetrahydrofuran overnight to dissolve the polystyrene, or by calcination to $500^{\circ} \mathrm{C}, 600^{\circ} \mathrm{C}, 800^{\circ} \mathrm{C}, 1000^{\circ} \mathrm{C}$, or $1100^{\circ} \mathrm{C}$ using a $2^{\circ} \mathrm{C} / \mathrm{min}$ heating and cooling rate and a two-hour hold, except for the case of $1100^{\circ} \mathrm{C}$, which was held at the final temperature for an extended five hours. A Lindberg Blue M box furnace from Thermo Scientific was used for calcination. Calcination temperatures below $500^{\circ} \mathrm{C}$ were not used to ensure complete removal of the polystyrene template. ${ }^{51}$ Deionized water was used from a Milli-Q system, ethanol was obtained from Koptec, and all other reagents (0.1M $\mathrm{HCl}$, styrene, tetraethylorthosilicate) were obtained from Sigma Aldrich.

Sample characterization. Scanning electron microscopy (SEM) was performed using a Zeiss Ultra Plus field emission SEM. ImageJ was used for subsequent image analysis including the width:height ratio calculations, based on the pixel size from the Zeiss SEM software.

X-ray photoelectron spectroscopy (XPS) was performed using a Thermo Scientific K-Alpha XPS system. Lower resolution survey scans were taken followed by higher resolution scans with $50 \mathrm{~ms}$ dwell time and $0.1 \mathrm{eV}$ resolution for the individual elements, with 10 scans averaged. The higher resolution scans were used for quantification. Attenuated total reflectance (ATR) infrared (IR) spectroscopy was performed using a Bruker Vertex 70 FTIR spectrometer equipped with a MIRacle attenuated total reflectance accessory. For each spot, 128 scans were averaged over the range $600-4000 \mathrm{~cm}^{-1}$ with $4 \mathrm{~cm}^{-1}$ resolution. 
For the optical measurements taken at normal incidence, a Leica DMRX microscope was used with an Ocean Optics USSB2000+ spectrometer. A $>96 \%$ reflective mirror was used as reference. Measurements of reference and sample spectra were performed under identical configurations, thus the data is displayed as absolute intensity. For the variable angle spectrometry studies, an Ocean Optics DH-2000 UV-VIS-NIR light source was used to illuminate a small spot $(3 \mathrm{~mm})$ of the sample at a given incidence angle. For each angle of illumination, light was collected at half degree increments for $-75^{\circ}$ to $+75^{\circ}$ relative to the sample normal and spectrally analyzed using an Ocean Optics Maya Pro 2000 spectrometer. A reference was taken with the detector at $180^{\circ}$ to the light source; however, the intensity data shown here is displayed in arbitrary units as the light source needed to be attenuated with a pinhole when acquiring the reference in order to prevent saturation of the detector. No quantitative color bar is shown, as only the spectral position of the reflectance bands is relevant for the argument made here.

Simulation. A 2D finite element simulation was carried out using the commercial software ABAQUS. A linear elastic material model was used. The shrinkage of the material was simulated as thermal strain. The displacement and rotation of the inverse opal structure proximate to the substrate were constrained, and a periodic boundary condition was applied on a unit cell in the lateral direction. The ratio of the pore width to the pore height was recorded as a function of the linear shrinkage.

\section{RESULTS AND DISCUSSION}

Anisotropic inverse opals were made using a slight modification to the synthesis procedure. ${ }^{11}$ The co-assembly of inverse opals involves one crystal growth step, in which a substrate (usually 
a silicon wafer) is submerged in a dispersion of polymeric colloids and pre-hydrolyzed tetraethyl orthosilicate (TEOS) in water. The water is evaporated at $65^{\circ} \mathrm{C}$, forming a thin polymer/silica opal film on the substrate. Next, the sample is calcined at $500^{\circ} \mathrm{C}$ in order to combust the colloids and sinter the sol-gel silica, leaving behind an ordered, porous silica structure. Changing this final calcination step results in an altered pore structure (Figure 1), giving access to different pore geometries. With spherical pore symmetry arising from the shape and size of the colloids, the as-grown structure can be obtained by dissolving the colloids with toluene or tetrahydrofuran (Figure 1A, left). By raising the calcination temperature, different degrees of anisotropy of the structure, with increasingly deformed pores, can be obtained (Figure 1A). Growing the matrix and template in a single, one-pot synthesis allows for large (cm-scale) domains,${ }^{11}$ and this high order is retained even at high calcination temperatures (Figure S1). Using the ratio of pore width and height to quantify the variation in the geometry, we see that the anisotropy introduced into the structure as a function of calcination temperature follows a roughly linear trend with temperature (Fig 1B). In all cases, the same heating rate $\left(2^{\circ} \mathrm{C} / \mathrm{min}\right)$ was used, and the final temperature was held for two hours, except for the case of $1100^{\circ} \mathrm{C}$, which was held for an extended five hours. 

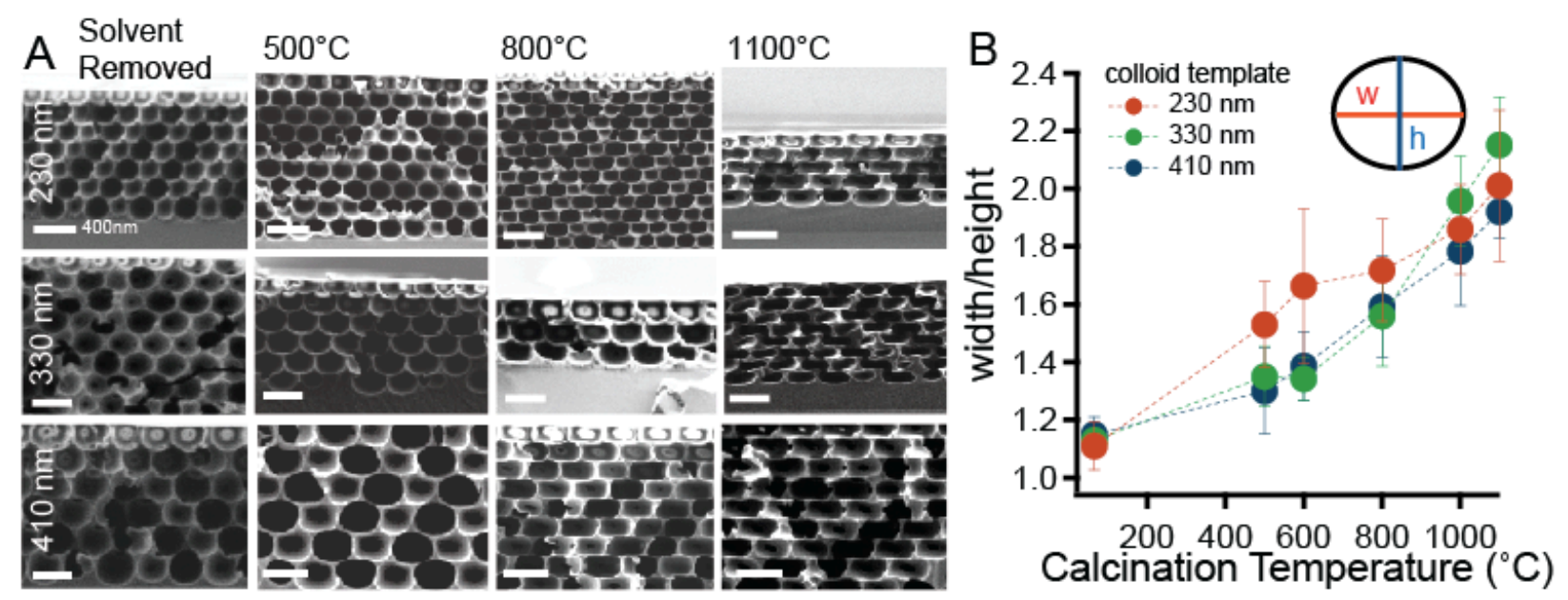

Figure 1. (A) Cross-sectional scanning electron microscope (SEM) images of samples made from 230, 330, and $410 \mathrm{~nm}$ polymer colloids as templates. Different samples are shown after template removal by solvent dissolution at room temperature (left) and by calcination at $500^{\circ} \mathrm{C}, 800^{\circ} \mathrm{C}$ and $1100^{\circ} \mathrm{C}$ (from left to right). All scale bars are $400 \mathrm{~nm}$. (B) Quantification of the anisotropy as a function of calcination temperature. The ratio of pore width (w) to pore height (h) for all samples was measured from the SEM images ( 25 measurements per sample). Error bars represent the standard deviation of these measurements assuming a Gaussian distribution.

Chemical Analysis of Si-OH Condensation. In order to determine what causes the observed structural changes on a molecular level, we used XPS and ATR-IR spectroscopy (Figure 2). XPS provides nanoscale surface-sensitive elemental information, whereas ATR-IR gives information on the chemical environment and probes farther into the structure. Together, the results allow us to probe the level of condensation at different calcination temperatures.

XPS confirms that silicon and oxygen are the only elements present in our silica film (Figure 2A). Further, the ratio of these two elements provides a way to monitor the condensation of the sol-gel silica: the $\mathrm{Si}$ :O ratio increases when two residual $\mathrm{Si}-\mathrm{OH}$ groups crosslink to form $\mathrm{Si}-\mathrm{O}-\mathrm{Si}$ and release a water molecule (Scheme 1). As shown in Figure 2B, we see an increase in the Si:O ratio with higher calcination temperatures, indicating that additional condensation is occurring. 
The ratio begins to level out at the value for a reference silica layer (horizontal dotted line) around $1000^{\circ} \mathrm{C}$.
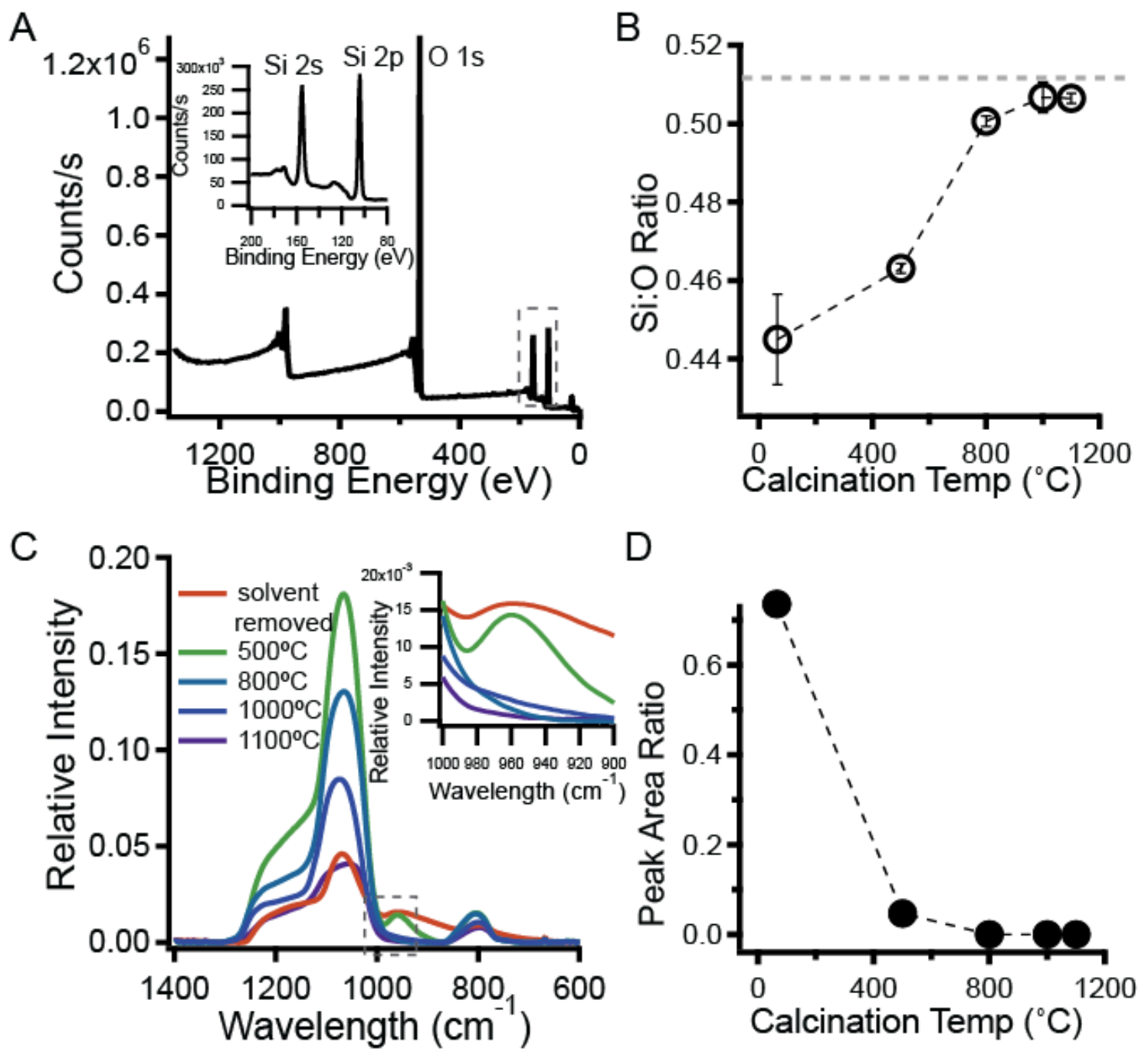

Figure 2. Analytical spectroscopy of the inverse opals. (A) XPS spectrum of a typical inverse opal sample, with the silicon region expanded in the inset. (B) An increase in the Si:O ratio with higher calcination temperatures is detected by comparing the peak areas. The horizontal dotted line represents a silicon oxide layer as reference. (C) ATR-IR vibrational spectra of the inverse opals, with the inset showing the $1000-900 \mathrm{~cm}^{-1}$ region characteristic of the $\mathrm{Si}-\mathrm{OH}$ stretch. (D) Graph of the area of the Si-OH peak $\left(970 \mathrm{~cm}^{-1}\right)$, normalized by the area of the $1070 \mathrm{~cm}^{-1} \mathrm{Si}-\mathrm{O}-\mathrm{Si}$ peak. 
This analysis is further confirmed with the ATR-IR spectra shown in Figure 2C. The peak at $970 \mathrm{~cm}^{-1}$, characteristic of the $\mathrm{Si}-\mathrm{OH}$ vibration, is shown in the inset. In order to better compare the peaks, the peak area at $970 \mathrm{~cm}^{-1}$ for each temperature is normalized by the peak area of the antisymmetric $\mathrm{Si}-\mathrm{O}-\mathrm{Si}$ vibration at $1070 \mathrm{~cm}^{-1}$ and shown in Figure 2D. The $\mathrm{Si}-\mathrm{OH}$ peak disappears at the higher calcination temperatures, indicating that $\mathrm{Si}-\mathrm{OH}$ bonds are no longer present and further confirming that condensation is occurring.

Shrinking of Inverse Opal Films. Condensation of silica sol-gels is known to cause shrinkage of the resulting silica. ${ }^{48-49}$ Since the silica inverse opals are confined in the lateral plane by the attachment to the silicon wafer substrate, volume reduction of the solid phase upon condensation causes shrinkage only in the direction perpendicular to the substrate (Figure 3A). To confirm that the substrate is preventing the matrix from shrinking in the lateral directions, we decoupled the inverse opal film from the substrate upon calcination using a thin layer of photoresist as a sacrificial layer deposited prior to the growth step. After assembly, this inverse opal film on a photoresist layer underwent calcination as before, and the sacrificial layer disintegrated with the colloids, causing the film to separate from the substrate (Figure 3B).

While films bound to a substrate show anisotropic pores (Figure 3C), the pores from samples with a sacrificial layer appear isotropic (Figure 3D). The anisotropy ratios of the pores from the bound films match those in Figure 1B, but the pores in the released films are the same size in both measured dimensions (Table 1). Comparing the $500^{\circ} \mathrm{C}$ sample and $1100^{\circ} \mathrm{C}$ samples in Table 1, the pores shrink $\sim 18 \%$ in each direction without a confining substrate, whereas the width stays the same but the height shrinks $\sim 42 \%$ for the substrate-bound sample. The pores of the unbound films shrink less in the vertical direction (18\% vs $42 \%)$, but they shrink in all directions equally, whereas all of the condensation-induced volume loss must be accommodated 
by the perpendicular direction on the substrate-bound films. In both cases, this corresponds to $\sim 40-45 \%$ volume loss, similar to what is seen in silica sol-gel films. ${ }^{48}$
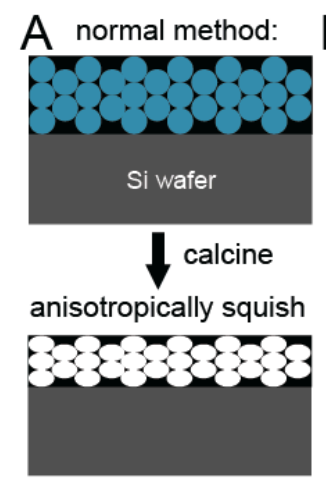

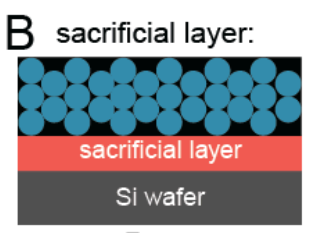

calcine isotropically shrink

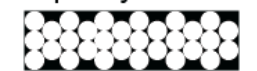

C normal method: $500^{\circ} \mathrm{C}$

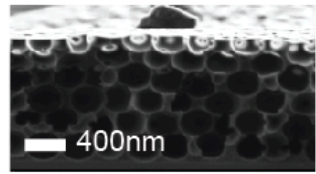

$1100^{\circ} \mathrm{C}$

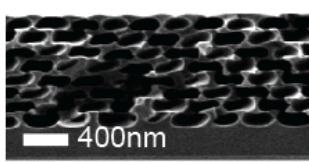

D sacrificial layer: $500^{\circ} \mathrm{C}$

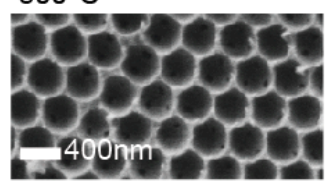

$1100^{\circ} \mathrm{C}$

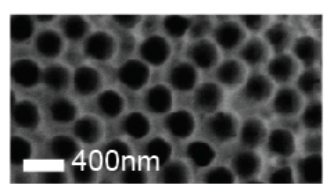

E simulation:

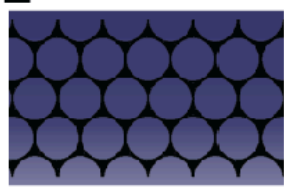

shrink

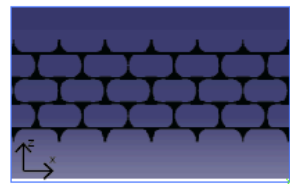

Figure 3. The effect of the substrate on pore anisotropy during calcination. (A-B) Schematics of the calcination step in inverse opals on a substrate (A), and with a sacrificial layer between the inverse opal and the substrate (B). (C-D) Cross-sectional SEM images of the samples attached to the substrate (C), and with a sacrificial photoresist layer (D), annealed to $500^{\circ} \mathrm{C}$ (top) and $1100^{\circ} \mathrm{C}$ (bottom). Scale bars are $400 \mathrm{~nm}$. (E) 2D theoretical model of structure change based on uniaxial shrinkage of the matrix in the vertical direction.

Table 1. Comparison of inverse opal pores with and without a substrate.

\begin{tabular}{|l|l|l|l|}
\hline Calcine Temp & $\mathbf{w}(\mathbf{n m})$ & $\mathbf{h}(\mathbf{n m})$ & w:h \\
\hline $500^{\circ} \mathrm{C}$ (substrate) & $320 \pm 14$ & $240 \pm 16$ & 1.33 \\
\hline $1100^{\circ} \mathrm{C}$ (substrate) & $330 \pm 14$ & $140 \pm 19$ & 2.36 \\
\hline $500^{\circ} \mathrm{C}$ (extra layer) & $330 \pm 15$ & $340 \pm 14$ & 0.97 \\
\hline $1100^{\circ} \mathrm{C}$ (extra layer) & $280 \pm 23$ & $270 \pm 17$ & 1.04 \\
\hline
\end{tabular}

The effect of the substrate is also visible in samples with cracks. When cracks occasionally form in inverse opals, the top layers become less confined along the substrate. The cracks can provide stress release sites in films annealed to high temperatures. Thus, while the bottom layers 
still deform perpendicular to the substrate, the top layers can now shrink more isotropically (Figure 4), creating a difference in pore dimensions between the upper and lower layers.

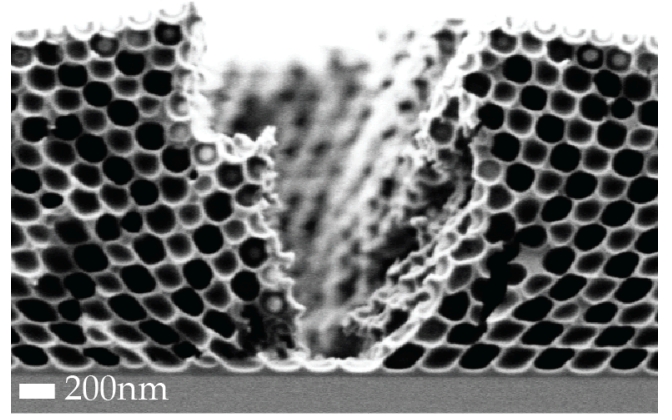

Figure 4. SEM image of a crack in an inverse opal made from a 230 nm colloidal template annealed to $800^{\circ} \mathrm{C}$.

To verify experimental data, the structural changes upon calcination were modeled using the commercial simulation software ABAQUS. A linear elastic material model was used in which the shrinkage was simulated as thermal strain. A 2D model of the pore structure was used with periodic boundary conditions in the lateral direction. Thus, the matrix height was shrunk without allowing any changes in the horizontal direction, similar to the schematic shown in Figure 3A. The results are presented in the supplemental movie, and images from two points in the video are shown in Figure 3E. The ratio of pore width to the pore height was recorded as a function of the linear shrinkage. The simulated shape qualitatively matches our experimental shape, indicating that the shape change is entirely due to matrix shrinkage upon continuing polycondensation.

The formation of the anisotropic structure is due to the shrinkage of the material while constrained on the bottom, so we expect a dependence on thickness. One way that this manifests itself is in edge effects. The size of the region influenced by the edge is comparable with the thickness of the film. In the middle part of the sample far away from the edges, the film is under homogenous deformation. As long as the lateral dimension of the sample is much larger than its thickness, there is always a region in the middle part of the sample where uniform opal structures are formed. Since the films were prepared over macroscopic dimensions, we expect a high 
homogeneity with little influence of sample edges. Previous studies found that cracks naturally form in co-assembled inverse opals once the film exceeds a thickness of approximately 20 colloid layers. ${ }^{11}$ As shown in Figure 4, such cracks prevent homogeneous pore shrinkage, and thus compromise the quality of the anisotropic film. Fortunately, large areas without cracks are possible with the co-assembly method. ${ }^{11}$ As long as the crack formation threshold is maintained, further annealing does not induce more cracking (Figure S1).

Table 2. Comparison of experimental and simulated anisotropic structures.

\begin{tabular}{|l|l|l|l|l|l|l|l|}
\hline \multicolumn{2}{|l|}{ Measured } & \multicolumn{2}{|l|}{ Simulated } \\
\hline $\begin{array}{l}\text { Calcination } \\
\text { Temperature }\end{array}$ & $\mathrm{w}(\mathrm{nm})$ & $\mathrm{h}(\mathrm{nm})$ & $\mathrm{w}: \mathrm{h}$ & $\%$ Shrinkage & $\mathrm{w}$ & $\mathrm{h}$ & $\mathrm{w}: \mathrm{h}$ \\
\hline $\begin{array}{l}65^{\circ} \mathrm{C}(\text { as } \\
\text { synthesized) }\end{array}$ & $370 \pm 16$ & $330 \pm 17$ & 1.12 & 3 & 1.00 & 0.89 & 1.12 \\
\hline $800^{\circ} \mathrm{C}$ & $420 \pm 33$ & $270 \pm 15$ & 1.56 & 10 & 1.01 & 0.67 & 1.5 \\
\hline $1100^{\circ} \mathrm{C}$ & $370 \pm 13$ & $190 \pm 10$ & 1.95 & 22 & 1.01 & 0.49 & 2.06 \\
\hline
\end{tabular}

The simulation and experimental results were compared using the ratio of the pore width to the pore height (Table 2). Specific values of the simulation $(3 \%, 10 \%$, and $22 \%$ shrinkage) were chosen to match the pore ratio to experimental examples $(1.12,1.5$, and 2$)$. Thus, we were able to correlate the experimental calcination temperature with the amount of contraction that caused the geometry shown in the simulation. Assuming both horizontal directions are equivalent in the three-dimensional experimental case, this gives an estimate that the silica matrix shrinks $40 \%$ in volume upon calcination at $1100^{\circ} \mathrm{C}$. This agrees with the one- and three-dimensional contraction values we see, as well as with previous results measuring shrinking of silica sol-gels. ${ }^{48-49}$ 
Optical properties of anisotropic inverse opals. The diameter of the templating colloidal particles was chosen to be several hundred nanometers, giving the inverse opals a periodic structure on the scale of the wavelength of visible light; thus, they form strongly colored photonic crystals. The perceived color of the films depends on several factors, including the number of layers in the inverse opal, the refractive index contrast between pore and matrix material, and, most importantly, the period of the repeating structure. ${ }^{5}$ Following Bragg's law, the layer spacing and the peak wavelength are directly proportional. The size of the colloidal building blocks and the calcination temperature (via the amount of shrinking) control this spacing. To minimize errors due to inter-sample variability, measurements of variations in the optical properties of inverse opal films as a function of preparation conditions were performed at the same spot on a single sample. The colloids were removed with solvent before measuring the reflectivity. The film then underwent calcination to subsequently higher temperatures, obtaining an optical spectrum after each heating step (Figure 5A). This experimental protocol differs slightly from the one used to obtain the samples from which previous results presented in this paper were deduced. However, similar pore anisotropies develop following this sequential protocol, and they are comparable to the anisotropies described above (Figure S2). We used 410 $\mathrm{nm}$ colloids as the colloidal template to ensure localization of the reflection peak across a range within the visible spectrum. 

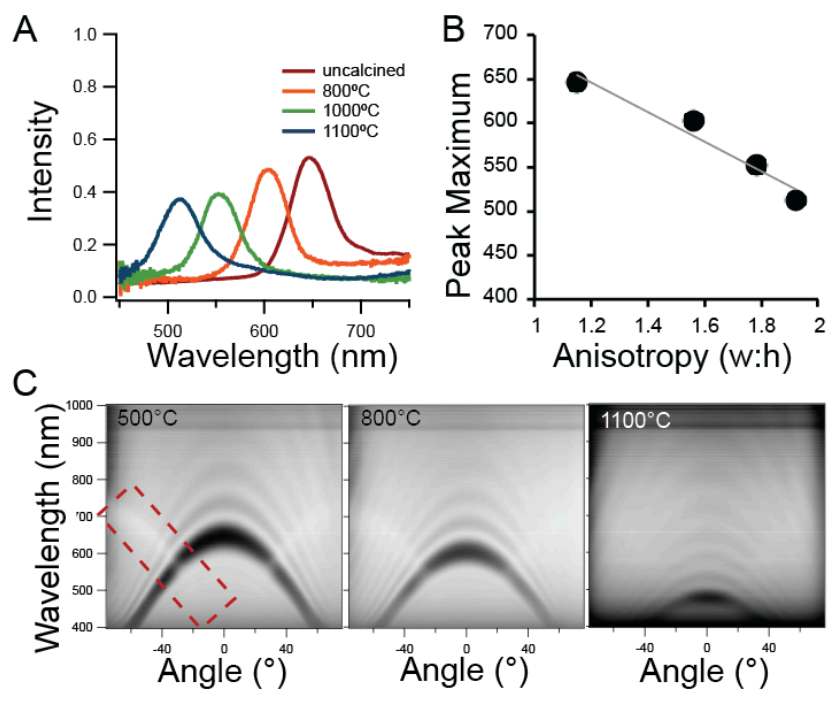

Figure 5. Calcination-induced anisotropy effects lead to variations in the optical properties of inverse opal films. (A) Optical spectra of a single sample calcined sequentially to higher temperatures. (B) Peak positions from (A) plotted against calcination temperature. (C) Effect of temperature on angular reflectance spectra.

The degree of anisotropy affects the periodicity and thus the wavelengths of Bragg resonance of light within the inverse opal structure. Consequently, annealing the same inverse opal sample to subsequently higher temperatures results in an incremental blue shift of the reflection peak (Figure 5A), originating from the decrease in period along the perpendicular direction. The shift in peak wavelength varied nearly linearly $\left(R^{2}=0.95\right)$ with anisotropy (Figure $5 \mathrm{~B}$ ), as expected from Bragg's law. The small deviation from linearity can be attributed to an expected increase in the refractive index from annealing, ${ }^{52}$ which would produce a red shift in the spectrum at higher temperatures. The peak intensity decreases slightly at elevated calcination temperatures, perhaps due to additional crack formation at higher temperatures due to condensation-induced stresses in the material. These cracks lead to a change in periodicity from the top layer to the bottom layer of the crystal, as shown in Figure 4. This leads to nonuniformity in pore geometry and thus layer thickness, resulting in peak broadening and lower reflection intensity.

The angular dependence of the optical spectra was also studied. Because the anisotropic shape of the inverse opal pores introduces a different periodicity as a function of incident angle, the angular dependence of the optical spectrum also changes when anisotropy is introduced. We 
obtained angularly resolved reflectance spectra to further probe the change in structure (Figure 5C). Specular reflectance spectra were obtained at incident angles from $-75^{\circ}$ to $75^{\circ}$ in $0.5^{\circ}$ increments with an angular resolution of less than $1^{\circ}$. These optical spectra were then plotted against the light incidence angle to give the graphs shown in Figure 5C, where the gray-scale encoded $($ black $=$ high, white $=$ low) reflection intensity is displayed as a function of light incidence angle and wavelength. The spectra qualitatively agree with other spectra in the literature. ${ }^{11,53}$ The undulations in the background of the spectra in Figure 5C and Figure 6 are due to thin film interference based on the thickness of the inverse opal film, which depends on the measurement method and is present because the light source probes a small area of uniform sample.

Different crystal planes present in the structure cause reflections at different angles. When they are not parallel to the substrate plane, they lead to dips in the angular spectra that appear as diagonal white lines (red box in Figure 5C). The presence of these dips indicates that the other crystal planes are still ordered enough to reflect light by constructive interference, indicating that a high degree of order remains in the samples, even those annealed to $1100^{\circ} \mathrm{C}$.

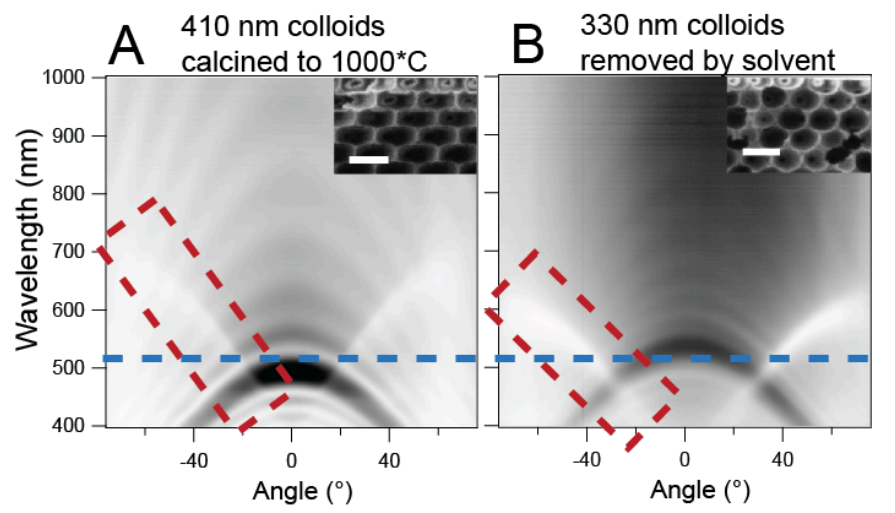

Figure 6. Comparison of angular reflection of inverse opals made from (A) $410 \mathrm{~nm}$ colloidal template removed by calcination to $1000^{\circ} \mathrm{C}$, and (B) $330 \mathrm{~nm}$ colloidal template removed by solvent. Insets are SEM images of the structures, with $400 \mathrm{~nm}$ scale bars.

To further probe the angular dependence, we compared inverse opals with the same normal reflection peak with two different degrees of anisotropy (Figure 6). In one sample, $410 \mathrm{~nm}$ 
colloids were used for the template, and calcination to $1000^{\circ} \mathrm{C}$ created anisotropy in the structure (Figure 6A). The periodicity of the anisotropic structure gave a normal reflection peak around $510 \mathrm{~nm}$ (lateral dashed line). A structure with similar periodicity will produce a similar wavelength of constructive interference, which can also be achieved by changing the colloid size. Thus, a second sample was made with $330 \mathrm{~nm}$ colloids as the template, and their removal with toluene left spherical pores (Figure 6B). This sample also has a normal reflection peak at $510 \mathrm{~nm}$. The biggest difference in the two samples is the angle of the dip in the spectra that appears as a white diagonal line (boxed). In the isotropic sample, the dip makes a much lower angle than the anisotropic sample on the right. This agrees with the fact that these crystal planes should be very different for the two samples. While changing the size of the colloids can control the normal reflection peak (although in a much more challenging way than simply altering the calcination temperature), it would not achieve the same angular dependence of the spectrum based on the different underlying geometry.

\section{CONCLUSIONS}

Anisotropic colloidal crystals have generated much interest recently. Here, we provide a method to controllably adjust the anisotropy present in an inorganic inverse opal matrix and describe the mechanism of formation. Controlling the shape of the pores by varying the calcination temperature gives us control over the order and porosity of the resulting film and introduces defined anisotropic pore shapes not easily achievable by direct assembly of anisotropic particles. Based on XPS and IR results, as well as a finite element simulation, we propose that matrix shrinkage from sol-gel condensation is causing this change in geometry. The decreased height of the pores leads to a blue shift in the optical spectrum at higher calcination 
temperatures, as expected for the smaller inter-layer spacing. A complete angular characterization of the optical spectra shows that three-dimensional order in the crystal is retained even with a high degree of anisotropy.

Calcination temperature can control the shape and anisotropy of inverse opal pores, allowing wide ranges of anisotropy perpendicular to the surface to be generated. In addition to the controlled tuning of inverse opals' optical properties described here, we expect anisotropy to affect other properties as well, such as the mechanical stability or wetting characteristics. This simple yet precise method for the control of anisotropy in three-dimensional periodic microstructures enables inverse opals to be a versatile platform for fundamental studies probing tailored confinements and structure-property relationships.

\section{ASSOCIATED CONTENT}

Supporting Information Available. Videos of structural simulation; volume loss calculations;

SEM cross-sections of samples after optics experiments. This material is available free of charge via the Internet at http://pubs.acs.org.

\section{AUTHOR INFORMATION}

\section{Corresponding Author}

*Address correspondence to jaiz@ seas.harvard.edu.

\section{Present Address}

"Department of Mechanical Engineering, Massachusetts Institute of Technology, Cambridge, MA, 02139 


\section{Notes}

The authors declare no competing financial interest.

Acknowledgement. K.R.P. acknowledges support from a National Science Foundation Graduate Research Fellowship and a National Defense Science and Engineering Graduate Fellowship from the Department of Defense. N.V. acknowledges funding from the Leopoldina Fellowship Program. M.K. acknowledges support from the Alexander von Humboldt Foundation. C.C.P. acknowledges the support of an Edward, Frances and Shirley B. Daniels and Wyss Fellowship while at the Radcliffe Institute for Advanced Study, 2012-2013. The authors thank Ian Burgess and Caitlin Howell for helpful discussions, and Grant England and Jack Alvarenga for help with the angular reflectance and infrared instruments, respectively. This work was funded with support from the Air Force Office of Scientific Research (AFOSR) under award number FA9550-09-0669-DOD35CAP (optical properties). This work was performed in part at the Center for Nanoscale Systems (CNS) at Harvard University supported by the National Science Foundation under NSF award no. ECS-0335765.

\section{REFERENCES}

(1) Whitesides, G. M. Science 2002, 295, 2418-2421.

(2) Mirkin, C. A.; Letsinger, R. L.; Mucic, R. C.; Storhoff, J. J. Nature 1996, 382, 607-609.

(3) Brinker, C. J.; Lu, Y.; Sellinger, A.; Fan, H. Adv. Mat. 1999, 11, 579-585.

(4) Cutler, J. I.; Auyeung, E.; Mirkin, C. A. JACS 2012, 134, 1376-1391.

(5) Galisteo-López, J. F.; Ibisate, M.; Sapienza, R.; Froufe-Pérez, L. S.; Blanco, Á.; López, C. Adv. Mat. 2010, 23, 30-69. 
(6) Vogel, N.; Goerres, S.; Landfester, K.; Weiss, C. K. Macromol. Chem. Phys. 2011, 212, $1719-1734$.

(7) O'Reilly, R. K.; Hawker, C. J.; Wooley, K. L. Chem. Soc. Rev. 2006, 35, 1068.

(8) Chen, Q.; Bae, S. C.; Granick, S. Nature 2011, 469, 381-384.

(9) Vogel, N.; Weiss, C. K.; Landfester, K. Soft Matter 2012.

(10) Sanders, J. Nature 1964, 204, 1151-1154.

(11) Hatton, B.; Mishchenko, L.; Davis, S.; Sandhage, K.; Aizenberg, J. PNAS 2010, 107, $10354-10359$.

(12) Freymann, von, G.; Kitaev, V.; Lotsch, B. V.; Ozin, G. A. Chem. Soc. Rev. 2013, 42, 2528.

(13) Arsenault, A. C.; Clark, T. J.; Freymann, von, G.; Cademartiri, L.; Sapienza, R.; Bertolotti, J.; Vekris, E.; Wong, S.; Kitaev, V.; Manners, I.; Wang, R. Z.; John, S.; Wiersma, D.; Ozin, G. A. Nat. Mater. 2006, 5, 179-184.

(14) Blanco, A.; Chomski, E.; Grabtchak, S.; Ibisate, M.; John, S.; Leonard, S.; Lopez, C.; Meseguer, F.; Miguez, H.; Mondia, J.; Ozin, G.; Toader, O.; van Driel HM Nature 2000, 405, $437-440$.

(15) Still, T.; Cheng, W.; Retsch, M.; Sainidou, R.; Wang, J.; Jonas, U.; Stefanou, N.; Fytas, G. Phys. Rev. Lett. 2008, 100, 194301.

(16) Guldin, S.; Hüttner, S.; Kolle, M.; Welland, M. E.; Müller-Buschbaum, P.; Friend, R. H.; Steiner, U.; Tétreault, N. Nano Letters 2010, 10, 2303-2309. 
(17) Tétreault, N.; Arsenault, E.; Heiniger, L.-P.; Soheilnia, N.; Brillet, J.; Moehl, T.; Zakeeruddin, S.; Ozin, G. A.; Grätzel, M. Nano Letters 2011, 11, 4579-4584.

(18) Lodahl, P.; Floris Van Driel, A.; Nikolaev, I. S.; Irman, A.; Overgaag, K.; Vanmaekelbergh, D.; Vos, W. L. Nature 2004, 430, 654-657.

(19) Cong, H.; Yu, B.; Wang, S.; Qi, L.; Wang, J.; Ma, Y. Optics Express 2013.

(20) Kolle, M.; Salgard-Cunha, P. M.; Scherer, M. R. J.; Huang, F.; Vukusic, P.; Mahajan, S.; Baumberg, J. J.; Steiner, U. Nature Nanotech. 2010, 5, 511-515.

(21) Petkovich, N. D.; Stein, A. Chem. Soc. Rev. 2013, 42, 3721-3739.

(22) Pikul, J. H.; Gang Zhang, H.; Cho, J.; Braun, P. V.; King, W. P. Nature Comm. 2013, 4, $1732-1735$.

(23) Esmanski, A.; Ozin, G. Adv. Funct. Mater. 2009.

(24) Lu, Y.; Yu, H.; Chen, S.; Quan, X.; Zhao, H. Environ. Sci. Technol. 2012, 46, 17241730.

(25) Chen, J. I. L.; Freymann, von, G.; Kitaev, V.; Ozin, G. A. JACS 2007, 129, 1196-1202.

(26) Burgess, I. B.; Mishchenko, L.; Hatton, B. D.; Kolle, M.; Lončar, M.; Aizenberg, J. JACS 2011, 133, 12430-12432.

(27) Choi, S.-W.; Xie, J.; Xia, Y. Adv. Mat. 2009, 21, 2997-3001.

(28) Vogel, N.; Belisle, R. A.; Hatton, B.; Wong, T.-S.; Aizenberg, J. Nature Comm. 2013, 4, $1-10$. 
(29) Raccis, R.; Nikoubashman, A.; Retsch, M.; Jonas, U.; Koynov, K.; Butt, H.-J.; Likos, C. N.; Fytas, G. ACS Nano 2011, 5, 4607-4616.

(30) Florez, L.; Herrmann, C.; Cramer, J. M.; Hauser, C. P.; Koynov, K.; Landfester, K.; Crespy, D.; Mailänder, V. Small 2012, 8, 2222-2230.

(31) Herrmann, C.; Turshatov, A.; Crespy, D. ACS Macro Lett. 2012, 1, 907-909.

(32) Champion, J. A.; Katare, Y. K.; Mitragotri, S. PNAS 2007, 104, 11901-11904.

(33) Jiang, P.; Bertone, J. F.; Colvin, V. L. Science 2001, 291, 453-457.

(34) Sacanna, S.; Korpics, M.; Rodriguez, K.; ndez, L. C. O. N.-M. E.; Kim, S.-H.; Pine, D. J.; Yi, G.-R. Nature Comm. 2012, 4, 1688-6.

(35) Sacanna, S.; Pine, D. J. Curr. Op. in Coll. \& Int. Sci. 2011, 16, 96-105.

(36) Mao, X.; Chen, Q; Granick, S. Nat. Mater. 2013, 12, 217-222.

(37) Wang, Y.; Wang, Y.; Breed, D. R.; Manoharan, V. N.; Feng, L.; Hollingsworth, A. D.; Weck, M.; Pine, D. J. Nature 2012, 490, 51-55.

(38) Yang, S.-M.; Kim, S.-H.; Lim, J.-M.; Yi, G.-R. J. Mater. Chem. 2008, 18, 2177.

(39) Jiang, X. C.; Zeng, Q. H.; Chen, C. Y.; Yu, A. B. J. Mater. Chem. 2011, 21, 16797.

(40) Glotzer, S. C.; Solomon, M. J. Nat Mater 2007, 6, 557-562.

(41) Alvarez-Puebla, R. A.; Agarwal, A.; Manna, P.; Khanal, B. P.; Aldeanueva-Potel, P.; Carbó-Argibay, E.; Pazos-Pérez, N.; Vigderman, L.; Zubarev, E. R.; Kotov, N. A. PNAS 2011, $108,8157-8161$. 
(42) Guerrero-Martínez, A.; Pérez-Juste, J.; Carbó-Argibay, E.; Tardajos, G.; Liz-Marzán, L. M. Angew. Chem. Int. Ed. 2009, 48, 9484-9488.

(43) Kuemin, C.; Nowack, L.; Bozano, L.; Spencer, N. D.; Wolf, H. Adv. Funct. Mater. 2011, $22,702-708$.

(44) Wei, W.; Chen, K.; Ge, G. Adv. Mat. 2013, 3863-3868.

(45) Ding, T.; Song, K.; Clays, K.; Tung, C.-H. Adv. Mat. 2009, 21, 1936-1940.

(46) Jones, M. R.; Macfarlane, R. J.; Lee, B.; Zhang, J.; Young, K. L.; Senesi, A. J.; Mirkin, C. A. Nat. Mater. 2010, 9, 913-917.

(47) Fu, M.; Chaudhary, K.; Lange, J.G.; Kim, H. S.; Juarez, J.J.; Lewis, J.A.; Braun, P.V. Advanced Materials, 2013. http://onlinelibrary.wiley.com/doi/10.1002/adma.201304809/abstract.

(48) Scherer, G. W. J. of Non-Crys. Sol. 1989, 109, 183-190.

(49) Hench, L. L.; West, J. K. Chem. Rev. 1990, 90, 33-72.

(50) Goodwin, J. W.; Hearn, J.; Ho, C. C.; Ottewill, R. H. Coll. and Polymer Sci. 1974, 252, 464-471.

(51) Peterson, J. D.; Vyazovkin, S.; Wight, C. A. Macro. Chem. and Phys. 2001, 202, 775784.

(52) Wang, X.; Wu, G.; Zhou, B.; Shen, J. Materials 2013, 6, 76-84.

(53) Guldin, S.; Hüttner, S.; Kolle, M.; Welland, M. E.; Müller-Buschbaum, P.; Friend, R. H.; Steiner, U.; Tétreault, N. Nano Letters 2010, 10, 2303-2309. 


\section{TOC GRAPHIC}

Isotropic inverse opal

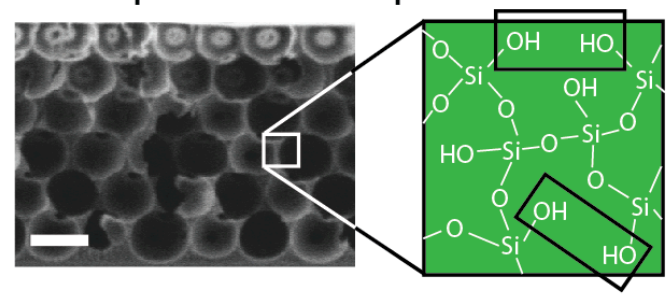

Anisotropic inverse opal

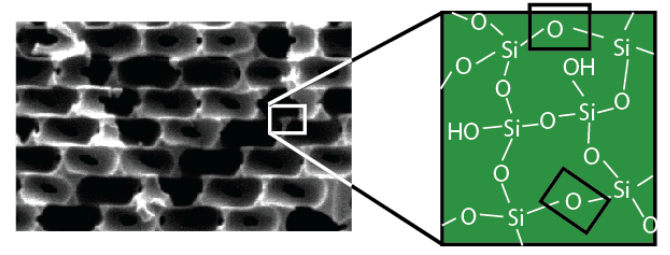

